\title{
PERVIVENCIA HISTÓRICA Y PROYECCIÓN SINTÁCTICA DE LA CONCINNITAS
}

\begin{abstract}
In this paper the author deals with the meaning of concinnitas in Cicero's works and also with the reasons for the disappearing of the term short after Cicero's death till its rehabilitation in Renaissance times. At the same time the author considers that the perception of concinnitas as a stylistic device can be most helpful to a good understanding of texts.
\end{abstract}

«...dass die concinnitas das am meisten Charakteristische der ciceronischen Diktion ist, lernt man schon auf der Schule...»'. Si esta caracteristica es incuestionable, como señala E. Norden, resulta, en cambio, paradójico que el término que la define no haya sobrevivido a su autor. Dicho término aflora exclusivamente en la edad madura del orador, cae prácticamente en el olvido tras su muerte y sólo en el Renacimiento volverá a ser rehabilitado.

$\mathrm{Si}$, como advierten G. Landgraf y E. Norden ${ }^{2}$, la redundantia iuuenilis de Cicerón se basaba en el empleo abusivo de la napíwoıs, паро-

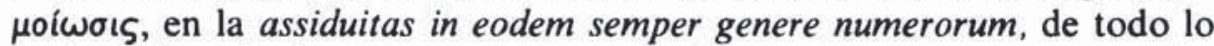
cual es ejemplo claro el Pro Quinctio o el Pro Sex. Roscio Amerino, las características más notables de sus discursos posteriores al viaje de es-

1 E. Norden, Die Antike Kunstprosa, Stuttgart 1958, p. 226.

2 G. Landgraf, Kommentar zu Ciceros Rede pro Sex. Roscio Amerino, Leipzig 1914 , p. 3: "Was nun unseren Cicero betrifft, so kann nicht geleugnet werden, dass in den beiden Erstlingsreden mancher rhetorische Aufputz, der zu den specifisch asianischen Effektmittels gehört, reichlich verwendet ist. Ich weise hiernur auf die

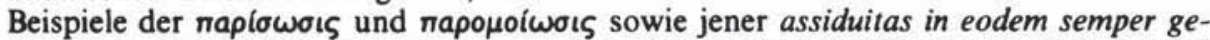
nere numerorum hin.» Norden, o.c., p. 227, caracteriza también este período juvenil por el uso abusivo de tales recursos: "Aber darauf ist noch nicht hingewiesen worden, dass er in seiner ersten Reden von diesem Redeschmuck einen ungehörigen Gebrauch macht, während er ihn später erheblich temperiert hat.» Tanto Landgraf como Norden toman como punto de partida los capítulos del Orat. 107 y 108 asi como el Brut. 316. 
tudios del 81 a. C. bien podrían ser la concinnitas y la uarietas. Precisamente, en correlato con este hecho estilístico el tratamiento de la concinnitas aparece sólo en sus estudios de madurez. Será, concretamente, en el Brut. 325 donde nos encontramos por primera vez con la referencia a este término: genera Asiaticae dictionis duo sunt: unum sententiosum et argutum, sententiis non tam crebris et seueris quam concinnis et uenustis... Aliud autem genus est non tam sententiis frequentatum quam uerbis uolucre atque incitatum... In his erat admirabilis orationis cursus, ornata sententiorum concinnitas non erat ${ }^{3}$. Con ello Cicerón estaba aludiendo a una de las características más diferenciadoras del estilo asiano a la que poco tiempo después señalará como fundamental para el ornatus oratorio. Sin duda las palabras de E. Norden sobre tal circunstancia son muy esclarecedoras ${ }^{4}$ : «En el proceso de formación de Cicerón como orador destaca el hecho de que él nunca se opuso frontalmente a la orientación asiana en el terreno estilistico. Compuso sus primeros discursos bajo la influencia del asiano Hortensio, luego se fue voluntariamente a Asia para estudiar el arte de la retórica en sus mismas fuentes. Nombra a todos aquellos maestros con respeto, pero a uno de ellos con respeto especial. A su regreso a Roma se sintió de nuevo hermanado literariamente con Hortensio, aun cuando, como él mismo afirma, la influencia moderadora de la escuela rodia habia recortado sus excesos estilísticos." Así pues, Cicerón sin renunciar a ciertos principios asianos sabrá dosificarlos y armonizarlos evitando, de este modo, los excesos de juventud y logrando un mayor equilibrio formal. Precisamente en el Orat. 175 hará referencia a la moderatio como característica que diferencia a Isócrates de sus predecesores Gorgias y Trasímaco, de quienes dice que hicieron un uso intemperantius de dichos recursos. Con esta comparación Cicerón estaba aludiendo a su etapa juvenil demasiado redundante frente al período de madurez. Concretamente en Orat. 39 el Arpinate había mostrado su identificación con Isócrates y su contraste con Gorgias, Trasímaco y Teodoro de Bizancio, señalando como defectos de éstos la búsqueda de un desarrollo demasiado versificado y el abandono de un período más rotundo. De igual modo en Orat. 107 contrastaba su iuuenilis redundantia con su madurez posterior, recordando con ello el contraste que había señalado entre Gorgias e Isócrates.

3 Cicerón habia presentado ya en Inu. 1, 18 el término concinnitudo: splendoris et festiuitatis et concinnitudinis minimum, propterea quod ex his suscipio quaedam apparationis atque artificiosae diligentiae nascitur. Tal vacilación entre formas en -tudo y -tas es la misma que nos ofrece con sanctitas y sanctitudo.

4 Norden, o.c., p. 225. 
Por otra parte Cicerón manifiesta su identificación con Demóstenes por su variedad estilística, variedad que, en cambio, no había alcanzado Hortensio, quien remanebat idem nec decebat idem (Brut. 327).

En definitiva, Cicerón se mostraba a sí mismo como la culminación de un proceso en el que la concinnitas de Isócrates y la uarietas de Demóstenes constituian las notas más preponderantes ${ }^{5}$.

Tales procedimientos literarios tan caros a Cicerón demandaban una formulación más explícita y concreta. Ésta terminó por abrirse camino: el término de concinnitas aparece ya definido en el capítulo 38 del Orator y a partir del capítulo 81 su presencia en este tratado es permanente, constituyéndose en la característica más señalada del período, esto es, del ornato literario ${ }^{6}$.

El hecho en sí ya importante adquiere mayor trascendencia al no figurar dicho término en el tratado De oratore escrito nueve años antes. En efecto, en dicho tratado señalaba el Arpinate como orationis laudem splendoremque ${ }^{7}$ aquella doble ornamentación: nam est quidam ornatus orationis, qui ex singulis uerbis est, alius qui ex continuatis coniunctisque constat $^{8}$. Dicho ornato estaba en función tanto de la palabra en sí portadora de valor literario como de la disposición artística de las palabras dentro del período. En el primer caso tal virtualidad literaria se conseguia por uno de estos tres procedimientos: Tria sunt igitur in uerbo simplici, quae orator adferat ad illustrandam atque exornandam orationem, aut inusitatum uerbum, aut nouatum, aut tralatum ${ }^{9}$. En el segundo caso tal virtualidad literaria se originaba mediante una adecuada disposición de los elementos del período y la consecución de un efecto rítmico: sequitur continuatio uerborum, quae duas res maxime, conlocationem primum, deinde modum quendam formamque desiderat ${ }^{10}$. Ambos aspectos se dirigían respectivamente al carácter eufónico y rítmico del período ".

Esta doble ornamentación requerida por el tratado De oratore constituía el presupuesto irrenunciable de todo discurso artístico, discurso que podía tomar luego las variantes estilísticas pretendidas por el orador gracias a las diferentes figuras de lengua y pensamiento: in perpetua

s Schanz-Hosius, Geschichte der römischen Literatur, Munich 1966, I, cap. 151: "Der Hohepunkt des Dialogs erscheint in dem Schluss, wo Cicero seinen eigenen Entwicklungsgang zeichnet und den Leser nicht im unklarem lässt, dass in ihm das Ideal des Redners verkörpert sei.»

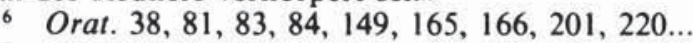

De orat. III 147.

De orat. III 149.

9 De orat. III 152 y 170.

10 De orat. III 171.

"De orat. III 171 y 173. 
autem oratione cum et coniunctionis leuitatem et numerorum, quam dixi, rationem tenuerimus, tum et quasi luminibus distinguenda et frequentanda omnis oratio sententiarum atque uerborum ${ }^{12}$.

Frente a este ornatus orationis ${ }^{13}$ desarrollado en el De oratore el Orator introduce la novedad antes indicada de la concinnitas. Así al hablar del ornatus Cicerón mantiene en este segundo tratado la división entre palabras consideradas aisladamente y su disposición artística en la frase ${ }^{14}$. Por otra parte, la división de palabras tomadas aisladamente respeta en esencia la anterior división ${ }^{15}$. Donde, por el contrario, estriba la gran diferencia entre uno y otro tratados ciceronianos es en la nueva formulación sobre la disposición de los elementos del período presentada en el segundo: ya no serán solamente dos aspectos los observados en este parámetro estilistico sino tres con la incorporación a los otros dos de la concinnitas, pues aunque en el De oratore está recogido este sentimiento por la simetria de la frase y aun cuando en el Orator nos encontramos con la sinonimia forma ipsa concinnitasque ${ }^{16}$ no aparece, en cambio, formulado de manera diferenciada. A ello conviene añadir los cambios terminológicos observados en este parámetro estilístico, reveladores, sin duda, de la nueva posición adoptada por Cicerón frente a este tema: Est enim in utroque et materia et tractatio: materia in uerbis, tractatio in collocatione uerborum. Ternae autem sunt utriusque partes: uerborum tralatum, nouum, priscum - nam de propriis nihil hoc loco dicimus -: collocatione autem eae, quas diximus, compositio, concinnitas, numerus ${ }^{17}$.

Así pues, el ornato literario deberá contar, según esta nueva formulación fijada en el Orator, con la concinnitas: collocata autem uerba habent ornatum si aliquid concinnitatis efficiunt ${ }^{18}$. También en el Orator la collocatio uerborum es considerada prioritaria a cualquier figura retórica. Concretamente Cicerón nos dice en esta obra después de haber hablado de las figuras de pensamiento y lengua lo siguiente: sed haec (sc. ornamenta), nisi collocata et quasi structa et nexa uerbis, ad eam laudem quam uolumus aspirare non possunt ${ }^{19}$. Tal disposición $\mathrm{u}$ ordenamiento (collocatio uerborum) deberá, pues, abarcar los tres aspectos antes señalados, cuyas características resume Cicerón de esta forma: collocabuntur

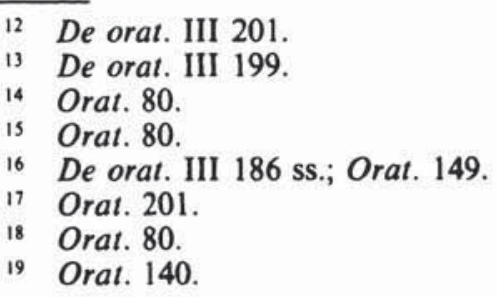


igitur uerba, aut ut inter se quam aptissime cohaereant extrema cum primis eaque sint quam suauissimis uocibus, aut ut forma ipsa concinnitasque uerborum conficiat orbem suum, aut ut comprehensio numerose et apte cadat $^{20}$.

Dicho recurso literario, aun cuando lo formuló Cicerón por primera vez con el término de concinnitas, tiene una historia que se remonta a Gorgias: in huius concinnitatis consectatione Gorgiam fuisse principem accepimus $^{21}$. Ahora bien, si este recurso literario, al menos en su esencia si no en su formulación, era ya antiguo, ¿cómo se explica que haya sido reconocido a última hora en el Orator? La cuestión, en nuestra opinión, responde al nivel de categorización de dicho recurso. Mientras en el tratado De oratore aparecen como figuras de lengua las llamadas figuras gorgianas, en el Orator dichas figuras continuarán teniendo su presencia en el catálogo de las figuras de lengua en cuanto no afectan a la totalidad del período ${ }^{22}$. Ahora bien, desde el momento en que dichas figuras son consideradas soporte estético necesario del período, entonces Cicerón les da otra categoría: tal es el caso de la concinnitas. Así quedaría resuelta una de esas contradicciones que suelen atribuírsele a esta obra ${ }^{23}$. Según A. Yon tal novedad estilística se debería al descubrimiento realizado a última hora por Cicerón sobre la vinculación de concinnitas y numerus: "Cicéron vient en effet de decouvrir l'interêt, pour ses recherches concernant le nombre, d'une méthode primitive dont il n'avait vu jusqu'alors que le caractère artificiel et quelque puéril» ${ }^{24}$. Efectivamente, a lo largo del Orator 164, 165, 202, 219, 220 el Arpinate señala dicho carácter rítmico, esto es, numerosus de la concinnitas, circunstancia ésta por la que la concinnitas se aproxima al concepto de numerus ${ }^{25}$. Por otra parte, se pueden observar ciertas expresiones referidas exclusivamente a la compositio y al numerus que parecen ignorar la competencia de la concinnitas ${ }^{26}$, así como la frecuente vinculación de concinnitas y numerus, dando pie con ello a la identificación de ambos aspectos ${ }^{27}$.

Ahora bien, el hecho de que la concinnitas genere un ritmo y pueda ser asociada, consiguientemente, al numerus no debe llevarnos a confundir ambas categorias. De ahí la advertencia clara y expresa que nos hace dicho autor en Orat. 182 sobre la diferencia de estos tres concep-

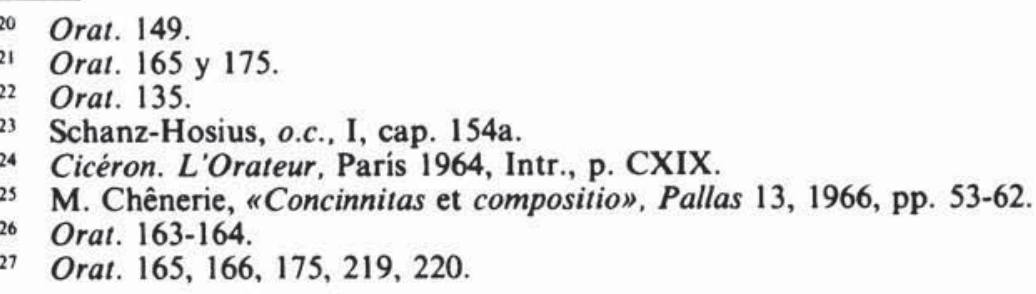


tos: at non est unum nec idem quod uoce iucundum est et quod moderatione absolutum et quod illuminatum genere uerborum: quamquam id quidem finitimum est numero quia per se plerumque perfectum est; compositio autem ab utroque differt, quae tota seruit grauitati uocum aut suauitati. De igual forma Cicerón al hablar del estilo humilde dice que aun cuando carezca de numerus ${ }^{28}$ no, por ello, deberá carecer de concinnitas $^{29}$.

$\mathrm{Si}$, pues, la concinnitas que se articulaba sobre las figuras gorgianas, esto es, sobre el isosilabismo, la adnominatio y la similicadencia de los miembros correspondientes, es lógico que el efecto rítmico fuera una consecuencia de la simetría. De ahí que al hacerse resaltar la correspondencia simétrica se incida inevitablemente en el efecto derivado que es el ritmo. Precisamente G. Devoto al fijarse en el carácter simétrico de la concinnitas apunta al efecto derivado de la misma cuando dice que su triunfo en la oratoria y literatura latinas se debió a la "predisposizione del latino da lunghisimo tempo all'ordinamento simmetrico, alla contraposizione degli elementi costitutivi della frase ${ }^{30}$. Ambos aspectos, simetría y ritmo tenían, pues, para Cicerón una relación de causa-efecto.

El origen de tal conflictividad sobre la naturaleza de la concinnitas y del numerus arranca, en nuestra opinión, del propio concepto de numerus y numerosus ampliamente desarrollado en el Orator ciceroniano y no siempre correctamente interpretado ${ }^{31}$. Mientras, unas veces, Cicerón al hablar de numerus se refiere únicamente al carácter ritmico, otras, precisa la naturaleza de este ritmo al señalar su vinculación a un ordo pedum $^{32}$. Consiguientemente, el numerus puede ser entendido genéricamente como ritmo, sin especificar su naturaleza o bien ser entendido específicamente en virtud de la oposición marcada por el término concinnitas; en este segundo caso numerus sería un ritmo cuantitativo determinado por un ordo pedum. Esta oposición nos explica, por otra parte, que la concinnitas sea, en opinión del Arpinate, generadora de un ritmo pero sin estar supeditada a un ordo pedum, requisito imprescindible, en cambio, para la formación de versos y cláusulas. De ahí que la concinnitas comparta con el numerus la atribución de numerosus, pero mientras en el caso de la concinnitas dicha naturaleza rítmica era libre, en el caso del numerus dependia totalmente de la naturaleza del pie ${ }^{33}$. Aquí se halla, pues, la clave que permite entender por qué en el De oratore

\footnotetext{
28 Orat. 77.

29 Orat. 83.

30 G. Devoto, Storia della lingua di Roma, Módena 1944 (reimpr. 1964), p. 162. 279-308.

C. Castillo, «Numerus, qui graece $\beta \cup \vartheta \mu \delta \varsigma$ dicitur», EMERITA 36, 1968, pp.

32 Orat. 227.

33 Orat. 67, 202, 219, 220.
} 
Cicerón estudiaba todavía la correspondencia de miembros e incisos bajo el capítulo del numerus ${ }^{34}$, aun cuando el propio autor era consciente de la diferencia entre un ritmo basado en ciertas correspondencias y aquel otro basado en la secuencia de pies: tal diferenciación la registra, en cambio, en el Orator. La novedad que ofrece este tratado es consiguientemente doble: en primer lugar Cicerón introduce el término de concinnitas para referirse al ritmo libre observado por las unidades correspondientes del período; en segundo lugar, incorpora a las llamadas figuras gorgianas a tal función rítmica del período ${ }^{35}$.

La evolución de estas categorías retóricas observadas en la obra retórica del Arpinate no la recogió Quintiliano, buen conocedor, no obstante, de estos tratados a los que alude constantemente. Tal circunstancia no encierra en sí mayor sorpresa, dado el doble comportamiento observado en aquellos dos tratados ciceronianos así como el doble estatuto de que gozaba la concinnitas en el Orator, puesto que Cicerón no dejó de considerarla como figura de lengua ${ }^{36}$. Ahora bien, lo que sí llama poderosamente la atención es el hecho de que Quintiliano haya aceptado, por un lado, la división tripartita del Orator y, por otro, el que haya modificado el contenido de tal división. Así, tras haber elogiado el estudio realizado por Cicerón sobre la composición del período y haber mostrado su fidelidad al maestro en este tema ${ }^{37}$ frente a las criticas vertidas por sus contemporáneos sobre esta doctrina ${ }^{38}$-entre estos críticos se hallaba precisamente Séneca el filósofo, cuya opinión sobre la concinnitas no podia ser más adversa ${ }^{39}$ - Quintiliano presenta la siguiente división relativa al ordenamiento artístico de la frase: In omni

\footnotetext{
34 De orat. III 186 y 190.

35 Orat. 37, 38, 39, 42, 65, 81, 83, 84, 149, 164, 165, 167, 181, 202, 219.

36 Orat. 81, 83, 181.

37 Inst. IX 4, 1.

38 Inst. IX 4, 3.

39 Séneca es continuador de la actitud estoica despectiva de todo ornamento oratorio y, consiguientemente, de la oratoria representada y defendida por el Arpinate: Epist. 114, 15: quorumdam non est compositio, modulatio est; adeo blanditur et molliter labitur. Quid de illa loquar in qua uerba differunt et diu exspectata uix ad clausulas redeunt? Quid illa in exitu lenta, qualis Ciceronis est, deuexa et molliter detinens nec aliter quam solet ad morem suum pedemque respondens? Esta crítica anticiceroniana se hará más fuerte en la epist. 115, 2: non est ornamentum uirile concinnitas. En Benef. VII 8, 2 Séneca hace una defensa de la oratoria estoica contraponiéndola a la ciceroniana: ... uirum exactae... sapientiae firmaeque in iis, quae proposuit, constantiae, eloquentiae uero eius, quae res fortissimas deceat, non concinnatae nec in uerba sollicitae sed ingenti animo, prout impetus tulit, res suas prosequentis.

Frente a tal actitud y como albacea de la estilistica ciceroniana se manifiesta Quintiliano, quien no repara en lanzar sus ataques contra los representantes del estilo estoico y, concretamente, contra su máximo representante de entonces, Séneca: Inst. X 1,125 ss.
} 
porro compositione tria sunt genera necessaria: ordo, iunctura, numerus ${ }^{40}$. Pocas líneas más adelante volverá a repetir el mismo esquema al hablar de los factores necesarios para obtener un discurso de calidad literaria: felicissimus sermo est, cui rectus ordo et apta iunctura et cum his numerus opportune cadens ${ }^{41}$.

Vemos, pues, que en principio Quintiliano respeta el mismo esquema tripartito presentado en el Orator, pero frente a esta adecuación formal se hallan notables diferencias tanto onomasiológicas como semasiológicas. En primer lugar, Quintiliano denomina compositio a lo que $\mathrm{Ci}$ cerón denominaba collocatio uerborum, denominación referida al concepto genérico, al carácter general de la disposición artística de los elementos de la frase. En segundo lugar, ni el término ni el concepto de ordo usados por Quintiliano encuentran correspondencia adecuada en el esquema del Orator. Por último, si el concepto del término iunctura empleado por Quintiliano viene a cubrir la misma parcela semántica que el de la compositio ciceroniana y si el término y concepto de numerus son superponibles en uno y otro autor, en cambio el término de concinnitas no tiene presencia en la división quintilianea ${ }^{42}$.

Quintiliano mantiene, como ya lo había hecho Cicerón siguiendo la tradición aristotélica ${ }^{43}$, no sólo la diferencia entre ritmo y metro ${ }^{44}$, sino también aquella misma diferencia observada por Cicerón entre numerus y numerosus ${ }^{45}, \mathrm{y}$, al igual que Cicerón lo había hecho en el De oratore, Quintiliano adscribe el carácter numerosus a los miembros e incisos conformadores del periodo ${ }^{46}$.

Se aparta, en cambio, del planteamiento del Orator al no aceptar el término de concinnitas y al reducir las figuras gorgianas - base de la concinnitas en el Orator - a un capitulo más de las figuras retóricas sin hacer mención al carácter numerosus que Cicerón les había reconocido en dicho tratado. Curiosamente Quintiliano usa como ilustración de dichas figuras aquellos mismos ejemplos de la oratoria ciceroniana que el Arpinate había presentado en el Orator para ilustrar la concinnitas. ¿Cómo se puede explicar esta ausencia de la concinnitas en un ciceroniano $\tan$ rendido como era Quintiliano? Ya hemos apuntado algunas razones tales como la evolución o cambio observado al respecto en el Orator frente al De oratore y el doble estatuto de que gozaba la concin-

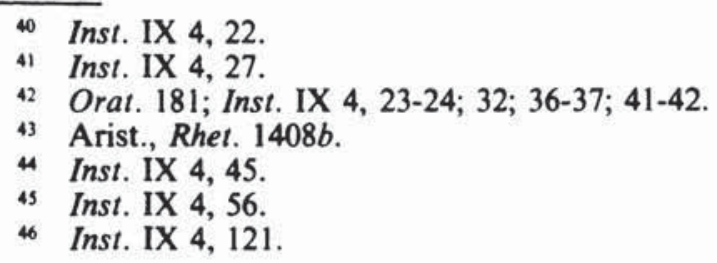


nitas en el Orator; de todos modos tales razones no son suficientes para pasar por alto un principio estético tan señero en el Orator ciceroniano. Creemos que Quintiliano no asumió dicho término tanto por las críticas de que era objeto entre sus contemporáneos como por la falsa identificación entre concinnitas y el tema concinere de la que él mismo fue vícti$\mathrm{ma}^{47}$ : si bien asumía el ritmo, recogido en el término numerus, no podía asumir el carácter melódico que la concinnitas le evocaba.

Conviene, pues, llamar la atención sobre este hecho para evitar los errores en que fácilmente se puede caer a la hora de comparar las formulaciones retóricas ofrecidas por Cicerón y Quintiliano ${ }^{48}$. Conviene advertir además que si en Cicerón la collocatio uerborum, como base del ornatus, era jerárquicamente anterior a las figuras retóricas, Quintiliano no comprende tal jerarquización posponiendo el estudio de la compositio al de las figuras.

Tal interpretación quintilianea había de tener no poca responsabilidad sobre la desaparición del término concinnitas en las obras de autores posteriores, dado el predicamento de que gozó la figura de aquél en el mundo latino ${ }^{49}$ : tanto el gramático Diomedes, como Fortunaciano, Julio Víctor, M. Capela, Isidoro, etc. ${ }^{50}$, siguen muy de cerca a Quinti-

47 La falsa etimologia de concinnus, concinnitas, concinnare sobre el tema canere había enraizado entre los escritores posteriores a Cicerón. Así el gramático Prisciano dirá (Gramm. III 467): concinnus... a canendo compositus est. Nonio nos da fe de tal confusión al decir (p. 43): recte autem concinnare et consentire intellegi potest quasi concinere. Responsable de tal error bien pudiera ser Varrón (L.L. VI 57: concinne loqui, dictum a concinere). Víctimas de esta falsa etimologia fueron precisamente Quintiliano y Séneca. De Quintiliano podemos presentar como prueba evidente la sustitución que ofrece de concinens por concinnus en el siguiente plagio de Cicerón:

De orat. III 203: reditus ad rem aptus et concinnus esse debebit. Inst. IX 1, 28: reditus ad rem aptus et concinens esse debebit.

De Séneca se deduce a través de epist. 114,15 y 115,2 la asociación de concinnitas con modulatio, esto es, con el tema de canere, como también del dial. VII 8, 5: Hoc modo una efficietur uis ac potestas concors sibi et ratio illa certa nascetur non dissidens nec haesitans in opinionibus comprensionibusque nec in persuasione, quae, cum se disposuit, et partibus suis consensit et ut ita dicam concinuit, summum bonum tetigit.

48 L. Rubio, Introducción a la Sintaxis Estructural del Latin, Barcelona 1982, pp. 197-198: el autor identifica la compositio de Cicerón con el ordo rectus de Quintiliano y la concinnitas ciceroniana con la iunctura quintilianea.

49 Schanz-Hosius, o.c., II, p. 486: «Die rhetoren natürlich konnten nicht an Quintilian vorübergehen." H. Rahn, Ausbildung des Redners, Darmstadt 1975, p. 826: "Er repräsentiert eine der einprägsamsten und wirkungsvollsten Lehrgestalten des Abendlandes." E. R. Curtius, Literatura europea y Edad Media latina, México 1955, p. 103: «Hacia el final de este periodo aparece la obra más extensa sobre la Retórica y la que mayor influencia va a tener.»

so Diom., Gramm. I 465, 23 ss.: componitur igitur et struitur omnis... oratio... in uerbis obseruandum est primum ne a maioribus ad minora descendat honoremque per- 
liano en este punto relativo a la ordenación artística de los elementos del período con el consiguiente silencio sobre la concinnitas ciceroniana. En efecto, el término genérico empleado por todos estos autores es el de compositio y no el de collocatio uerborum; por otra parte, el término específico de iunctura ha desbancado totalmente al de compositio ciceroniano; en fin, ninguno de estos autores utiliza ya el término de concinnitas. Tal situación habría de ser modificada con el Renacimiento.

Como ya habiamos indicado, el Humanismo restaura para los estudios de retórica el término y el concepto de concinnitas. Figuras tan representativas del Humanismo español como las del Brocense, Luis Vives, García Matamoros, Lorenzo Palmireno, Fox Morcillo, etc., introducen en sus estudios de retórica el término de concinnitas. Conviene señalar, al respecto, la denuncia de Palmireno sobre la interpretación de Quintiliano con relación al Orator ciceroniano ${ }^{51}$, pero lo que resulta especialmente interesante es la interpretación del concepto de concinnitas ofrecida por el Brocense: collocatio quoque rerum appellatur dispositio in uerbis uero numerus siue concinnitas ${ }^{52}$. Según esta interpretación el Brocense parece identificar dicho concepto con el de numerus; ahora bien, para evitar equívocos entre ambos términos establece a renglón seguido la diferencia entre numerus naturalis o concinnitas y numerus artificiosus o rythmus. Esta interpretación original contrasta con aquellas otras dadas por humanistas más fieles tal vez a la letra que al pensamiento de Cicerón. En efecto, cuando leemos a Luis Vives o a Fox Morcillo nos irrita el que ambos humanistas confundan los términos y conceptos de

\footnotetext{
dat oratio... cauendumque ne fortiori coniungetur infirmius, ut sacrilego fur, homicidae corruptor... Sit et illa naturalis obseruatio, ne iunctura uerborum cacenphaton sonet...; Fortun., Rhet. 3, 4, p. 127 ss.: Quae in structura obseruanda sunt? ut frequentior sit rotunda quam plana; ne hiulca sit uocalium et maxime longarum crebra concursione... qui est compositionis ordo? Vt anteponamus genera quam species...; ut uerbis plerumque in conclusione quam nominibus utamur...; Iul. Vict., Rhet. 20, p. 432: Sequitur continuatio uerborum, quae duas res maxime, collocationem primum, deinde modum quendam formamque desiderat...; ne sit hiulca uocalium et maxime longarum crebra conclusione...; Mart. Cap., V 514: iam compositionis praecepta percurram, cuius uitium maximum est hiulcas et asperas...; 518: uitandum etiam cacemphaton uel interpositione uel conmutatione uerborum...; 519: his breuiter intimatis pedes sunt asserendi, quibus clausulae decenter aptentur; Isid., Orig. II 18, 1: componitur autem instruiturque omnis oratio...; $(19,2)$ in litteris, ut iunctura apta et conueniens sit...; $(20,2)$ fugienda etiam hyperbata longiora...

${ }_{51}$ Laurenti Palmireni de uera et facili imitatione Ciceronis cui aliquot opuscula studiosis adolescentibus utilissima adiuncta sunt..., Caesaraugustae 1560, f. $75^{\text {v: }}$ Fabius Quintilianus diuersam rationem sequitur, inquit, enim in omni compositione desiderantur tria, ordo, iunctura et numerus. Sed ut uideo ordinem et dispositionem confundit...

s2 De arte dicendi, ed. Mayáns, 1765, I, pp. 305, 360 ss.
} 
concinnitas y concentus $^{53}$ - creaciones ambas ciceronianas de valor diferente - reflejando, de este modo, aquella misma confusión generada entre los autores latinos posteriores a Cicerón, especialmente los gramáticos. Ahora bien, mayor extrañeza produce la definición que García Matamoros nos ofrece del término concinnitas cuando dice: concinnitas uero est, quum paria paribus relata relatis respondent ${ }^{54}$. Tal definición incomprensible es el producto centonario de una contaminación de dos definiciones dadas por Cicerón ${ }^{55}$.

Sea como fuere, al Humanismo le debemos la rehabilitación del término cuya vida apenas había superado en el mundo romano a la de su creador, pero cuyos efectos demandan una merecida atención.

Así lo hizo ver muy acertadamente G. Devoto al señalar el carácter de disciplina sintáctica que tenía la concinnitas, basándose para ello en ciertas irregularidades sintácticas imputables exclusivamente a aquélla ${ }^{56}$. Y si desde este punto de vista G. Devoto explica ciertos hechos de sintaxis extraños a la norma latina, L. Laurand se servirá de la misma para obtener la fijación de un texto latino transmitido con distintas variantes ${ }^{57}$.

Nosotros creemos que su virtualidad no se agota en estos dos aspectos. En nuestra opinión, la detectación de dicho recurso literario puede ser en determinadas situaciones un criterio conveniente para resolver algunas dificultades sintácticas y, en consecuencia, de interpretación. Por otra parte, creemos que esta posibilidad de interpretación sintáctica no tiene que limitarse a la obra de Cicerón sino que puede aplicarse a cuantos hicieron uso de tal recurso estilístico.

Para verificar cuanto estamos diciendo hemos considerado oportuno ofrecer dos textos en los que la detectación de este procedimiento literario puede coadyuvar a la mejor comprensión de los mismos.

Itaque ut ego, cum a nostro Catone laudabar, uel reprehendi me a ceteris facile patiebar, sic Isocrates uidetur testimonio Platonis aliorum iudicia debere contemnere (Orat. 41).

${ }_{33}$ Ioannis Lodouici Viuis Valentini de disciplinis libri $X X$, Antuerpiae 1531, f. 52r; Sebastiani Foxii Morzilli Hispalensis de imitatione seu de informandi styli ratione libri II, Antuerpiae 1554 , ff. $51^{\vee}$ y $62^{\mathrm{v}}$.

44 De tribus dicendi generibus siue de recta informandi stili ratione commentarius, en Opera omnia, Madrid, Andrés Ramirez, 1769, p. 487.

ss Cic., Orat. 38 y 84.

s6 Devoto, o.c., p. 163.

57 Laurand, Étude sur le style des discours de Cicéron, Amsterdam 1965, I, p. 123. 
Este período o unidad superior está formado por dos miembros o unidades mayores y, a su vez, cada miembro está formado por dos incisos o unidades menores. Si reparamos en que cada uno de los incisos de un miembro guarda correlación con el inciso correspondiente del otro miembro obtenemos fácilmente la clave interpretativa del texto. Es decir, si nos damos cuenta de que el inciso cum a nostro Catone laudabar tiene como correspondiente el inciso testimonio Platonis y que el segundo inciso del primer miembro uel reprehendi me a ceteris facile patiebar se corresponde al segundo inciso del segundo miembro aliorum iudicia debere contemnere, la interpretación del texto ya no puede ofrecer duda alguna.

Este método nos permite, por tanto, corregir algunas traducciones como la ofrecida por J. M. Casas i Homs en la versión al catalán de la Institutio oratoria de Quintiliano en la colección Bernat Metge.

Sed sicut firmiores in litteris profectus alit aemulatio, ita incipientibus atque adhuc teneris condiscipulorum quam praeceptoris iucundior hoc ipso quod facilior imitatio est (I 2, 26).

La traducción catalana es la siguiente: «Però aixi com l'emulació afavereix els progressos dels qui han avançat més en l'estudi, semblantment als qui comencen i fins als més petits dels condeixebles els plau més la imitació dels grans que la dels mestres, perquè s'els fa més fàcil.» También en este texto latino hay unas correlaciones entre las distintas unidades del periodo que el traductor no ha recogido: observamos la primera correspondencia entre firmiores in litteris del primer miembro e incipientibus et adhuc teneris del segundo; la segunda correspondencia no observada por el traductor es la que hay entre profectus... aemulatio y condiscipulorum... imitatio. La traducción debería ser, en consecuencia, la siguiente: "Así como a los más aventajados en los estudios les estimula la emulación del éxito, así a los que se inician les es más grata por el simple hecho de que les es más fácil la imitación de sus condiscipulos que la del preceptor.»

Creemos, pues, que este recurso estilístico tan grato a Cicerón encierra una gran virtualidad hermenéutica especialmente en aquellos textos de mayor complejidad, como advierte G. Devoto. Por otra parte, conviene advertir que desde esta perspectiva de la concinnitas encuentran mejor explicación las unidades menores del período, esto es, miembro e inciso, tan confusamente explicadas en los manuales de retórica ${ }^{58}$.

\footnotetext{
58 H. Lausberg, Manual de retórica literaria, Madrid 1966.
} 
En definitiva, el reconocimiento de este recurso literario de la concinnitas, fijado onomasiológica y semánticamente por Cicerón, ignorado, en cambio, por Quintiliano y generaciones posteriores hasta su rehabilitación con los Humanistas, puede servirnos de instrumento valioso para la interpretación de los textos latinos allí donde aquélla se produzca.

Antonio Alberte González 